\title{
Live Observation of Atherosclerotic Plaque Disruption in Apolipoprotein E-Deficient Mouse
}

\author{
Authors \\ V. Daeichin ${ }^{1}$, J. C. Sluimer ${ }^{2}$, K. van der Heiden ${ }^{1}$, I. Skachkov ${ }^{1}$, K. Kooiman ${ }^{1}$, A. Janssen ${ }^{2}$, B. Janssen ${ }^{3}$, \\ J. G. Bosch , N. de Jong ${ }^{1,4}$, M. J. A. P. Daemen ${ }^{5}$, A. F. W. van der Steen ${ }^{1}$ \\ Affiliations \\ Affiliation addresses are listed at the end of the article
}

Key words
ultrasound
microbubbles
plaque rupture
ApoE
atherosclerosis

received $\quad 15.07 .2015$

accepted $\quad 04.10 .2015$

Bibliography

DOI http://dx.doi.org/

$10.1055 / \mathrm{s}-0035-1565092$

Ultrasound International Open

2015; 1: E67-E71

(c) Georg Thieme Verlag KG

Stuttgart · New York

ISSN 2199-7152

Correspondence

Dr. Verya Daeichin, PhD

Erasmus Medical Center

Thoraxcenter Biomedical

Engineering

Dr. Molewaterplein 50

3015 GE Rotterdam

Netherlands

Tel.: + 31/10/704 4638

Fax: $+31 / 10 / 7044720$

v.daeichin@erasmusmc.nl

\begin{abstract}
$\nabla$

Aim: The actual occurrence of spontaneous plaque rupture in mice has been a matter of debate. We report on an in vivo observation of the actual event of possible plaque disruption in a living $\mathrm{ApoE}^{-/-}$mouse.

Methods and Results: During live contrastenhanced ultrasonography of a 50-week-old $\mathrm{ApoE}^{-1-}$ male mouse, symptoms suggesting plaque disruption in the brachiocephalic artery were observed. Histological analysis confirmed
\end{abstract}

\section{Introduction}

$\nabla$

Atherosclerosis is a lipid- and inflammationdriven disease of the arteries that leads to development of plaques. Plaque disruption, either by rupture of the fibrous cap or dissection of the plaque from the tunica media $[1,2]$, is the main cause of cardiovascular events, such as stroke and myocardial infarction $[3,4]$. While human plaque histopathology provides valuable data on plaque disruption $[3,5,6]$, there is an urgent need for representative animal models in which prospective examination of the events leading up to plaque disruption, the disruption itself, and the effect of plaque stabilizing therapies can be studied. The most abundantly used animal model to study atherosclerosis is the mouse. However, whether plaque disruption actually occurs in mice has been a matter of debate in the past decade [7-13]. Features suggestive of plaque rupture in apolipoprotein E-deficient $\left(\mathrm{ApoE}^{--}\right)$mice with spontaneously developed unstable atherosclerosis in the brachiocephalic artery have been reported [14-19]. However, these were criticized by Schwartz et al. [7], who suggested that the reported findings may actually be sectioning artifacts, intraplaque hemorrhage, or a completely different form of lesion disruption not seen in human plaques. Furthermore, the inter- the presence of advanced atherosclerotic lesions with dissections and intraplaque hemorrhage in the affected brachiocephalic trunk, pointing towards plaque rupture as the cause of the observed event. However, we did not detect a luminal thrombus or cap rupture, which is a key criterion for plaque rupture in human atherosclerosis.

Conclusion: This study reports the real-time occurrence of a possible plaque rupture in a living $\mathrm{ApoE}^{-/-}$mouse. 
air at the rate of 160 strokes per minute. The left jugular vein was exposed, and a heat-stretched polyethylene- 25 cannula was inserted $(1.5 \mathrm{~cm})$ and subcutaneously guided to the neck of the mouse. Here the catheter was fixed, extended, filled with heparinized saline $(10 \mathrm{U} / \mathrm{ml})$, and plugged. The animal was placed on the Vevo Rail System heating stage (VisualSonics Inc. Toronto, Canada) and ultrasound imaging was performed. A series of injections were done using a programmable syringe pump (11 Pico Plus Elite, Harvard Apparatus, Kent, UK) at a rate of $600 \mu \mathrm{L}$ per minute. The interval between ultrasound contrast agent (UCA) injections ( $100 \mu \mathrm{L}$ per injection) was $20 \mathrm{~min}$ to prevent a sudden increase in blood pressure due to volume overload. All animal work was approved by the regulatory authorities of Maastricht University and performed in compliance with the Dutch government guidelines.

\section{Ultrasound imaging}

UCA was prepared from Target-Ready Vevo MicroMarker contrast agent (VisualSonics Inc. Toronto, Canada) according to the manufacturer's instruction. Microbubbles were targeted with biotinylated antibodies against $\alpha_{v} \beta_{3}$-integrin (cat. no. 551380, BD Biosciences, San Jose, CA, USA) for making targeted UCA. An inactive form of biotinylated $\operatorname{IgG}(\mathrm{K})$ (cat. no. 553923, BD Bio- sciences) was used for making control UCA. In order to check the specific binding of the targeted UCA to the $\alpha v \beta 3$-integrin receptors in the animals, pure antibodies ( $200 \mu$ with concentration of $0.5 \mathrm{mg} / \mathrm{mL}$, cat. no. 550024, BD Biosciences) were injected to block the receptors after injection of the first targeted UCA. Then, another injection of the targeted UCA was performed. A high frequency pre-clinical ultrasound scanner, with a 256-element linear array transducer (Vevo 2100 with MS250 probe, VisualSonics Inc., Toronto, ON, Canada) was operated at $18 \mathrm{MHz}$. The system was operated at $10 \%$ transmit power in the contrast mode with a wide beam width, resulting in low and uniform transmit pressure over the imaging depth $(\sim 400 \mathrm{kPa}$, MI of 0.09). Side-by-side B-mode and nonlinear contrast mode (Nonlinear Fundamental [21]) images were acquired with a frame rate of 20 frames per second. The field of view was $11 \mathrm{~mm}$ deep and $17 \mathrm{~mm}$ wide. The focus of the transducer was set at $7 \mathrm{~mm}$ where the upper wall of the right common carotid was located. In the field of view, part of the aortic arch, the right brachiocephalic trunk, the right common carotid artery and its bifurcation were visible. In the imaging field, the right salivary gland was located on top of the right carotid. The B-mode gain was $20 \mathrm{~dB}$ and the contrast gain was $30 \mathrm{~dB}$. DICOM (Digital Imaging and Communications in Medicine) images were exported to MAT-
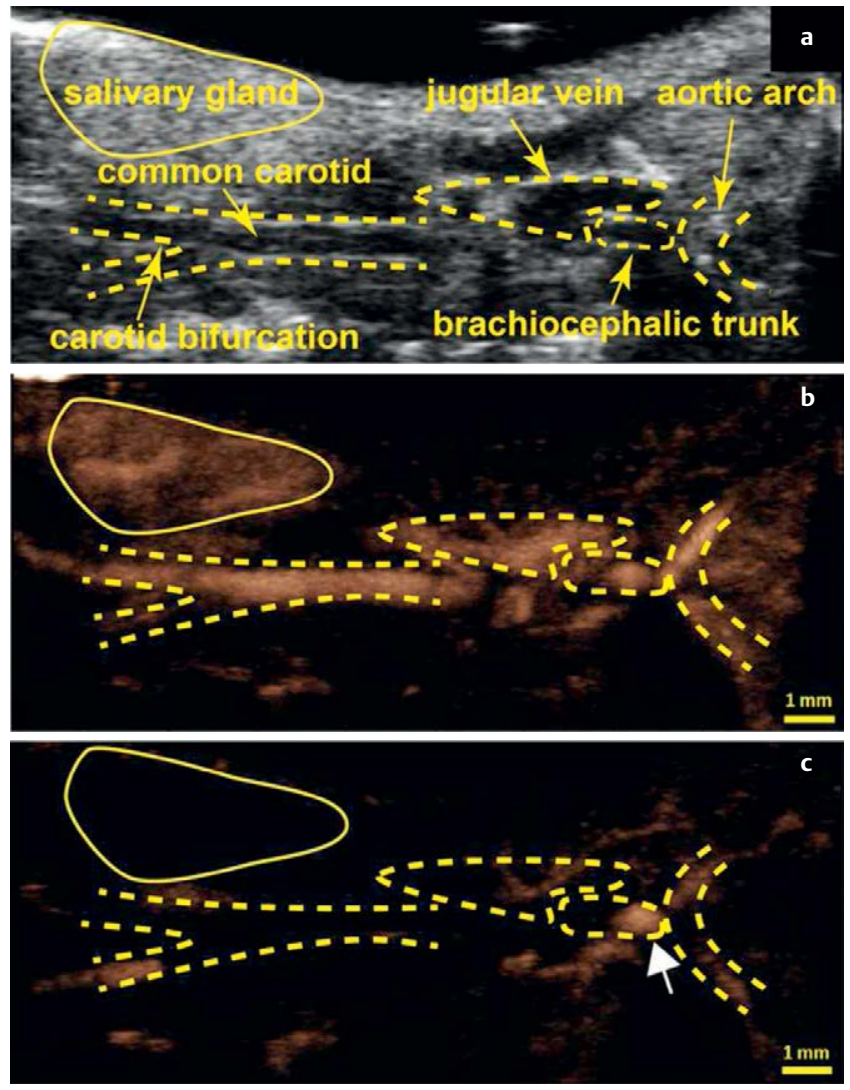

Fig. 1 Dynamics of plaque disruption imaged with contrast-enhanced ultrasound a B-mode image of part of aortic arch, beginning of brachiocephalic trunk, right common carotid, right carotid bifurcation (dashed lines) and right salivary gland (solid lines) of the $\mathrm{ApoE}^{-/-}$mouse. $\mathbf{b}$ Maximum Intensity Projection (MIP) over 100 frames (5s) after arrival of UCA in the aortic arch (first UCA injection) showing the trajectory of the aortic arch, right carotid artery, and the perfused salivary gland. c MIP over 100 frames (5s) after arrival of UCA in the aortic arch (third UCA injection) showing the accumulation of UCA in beginning of brachiocephalic trunk (white arrow)
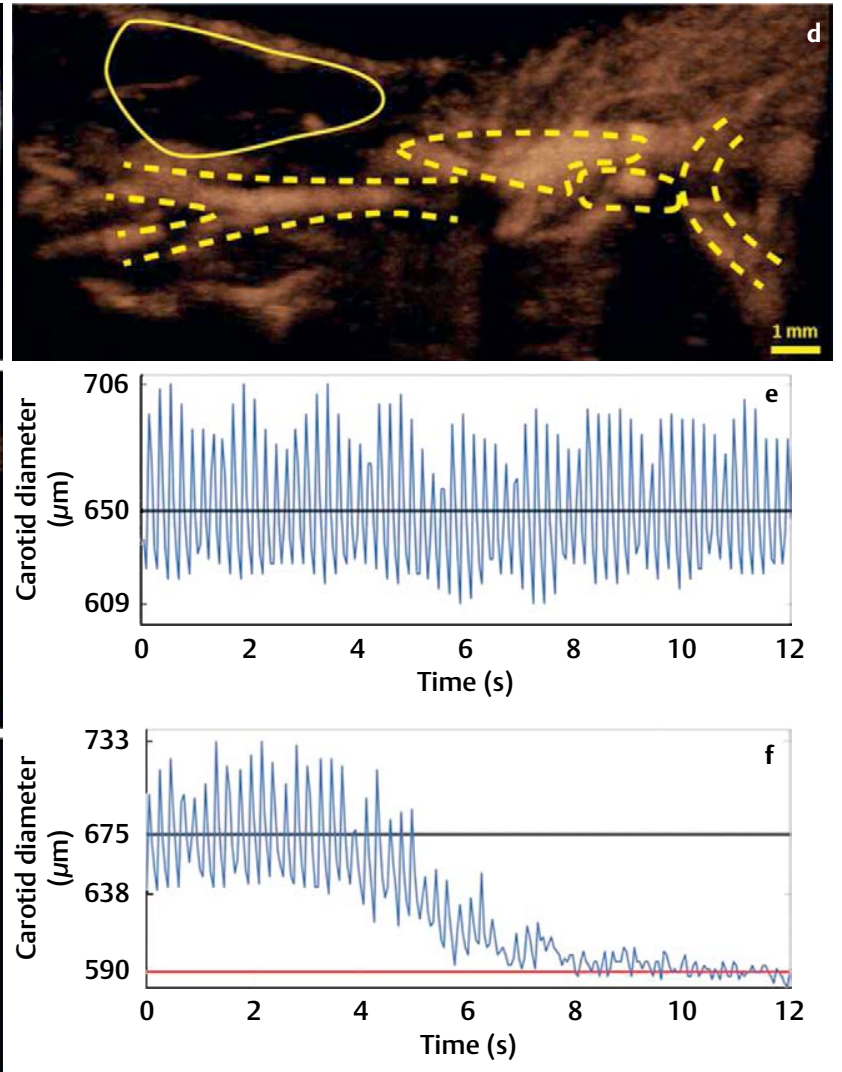

and significant reduction of the blood flow in the right carotid artery and no perfusion of the UCA in the salivary gland. $\mathbf{d}$ Longer MIP of the sequence in $\mathbf{c}(15 \mathrm{~s})$ showing the return of the UCA in the right common carotid but still no perfusion of the UCA in the salivary gland. e Normal pulsation of the right common carotid walls (subtraction of upper and lower wall displacement) before plaque disruption. $\mathbf{f}$ Pulsation of the right common carotid walls during plaque disruption showing the decrease in right common carotid pulsation followed by vessel constriction. 
LAB (Ver. R2012b, The MathWorks, Natick, MA, USA) for further off-line processing.

Tissue collection and histology

At sacrifice blood was drawn from the right ventricular apex. Subsequently, the mouse was gently perfusion fixed via the left cardiac ventricle with sodium nitroprusside $(0.1 \mathrm{mg} / \mathrm{ml}$; SigmaAldrich, Seelze, Germany), followed by $1 \%$ paraformaldehyde (PFA). The right carotid artery including the brachiocephalic artery was excised, fixed in $1 \%$ PFA for $24 \mathrm{~h}$, and paraffin-embedded. The right carotid artery was serially sectioned $(4 \mu \mathrm{m})$, and stained at $20 \mu \mathrm{m}$ intervals with Hematoxylin and Eosin (HE,
Sigma-Aldrich) [22] for microscopic examination by an experienced cardiovascular pathologist (M.D.). Serial sections were stained with the Martius Scarlet Blue (MSB) method to detect fibrin/fibrinogen as bright red, and Perl's Prussian Blue for Ferric Iron [22].

\section{Results}

$\nabla$

- Fig. 1a shows the anatomical structure of the animal in the ultrasound B-mode image. No physiological abnormalities were observed in the animal during the injections of the first control
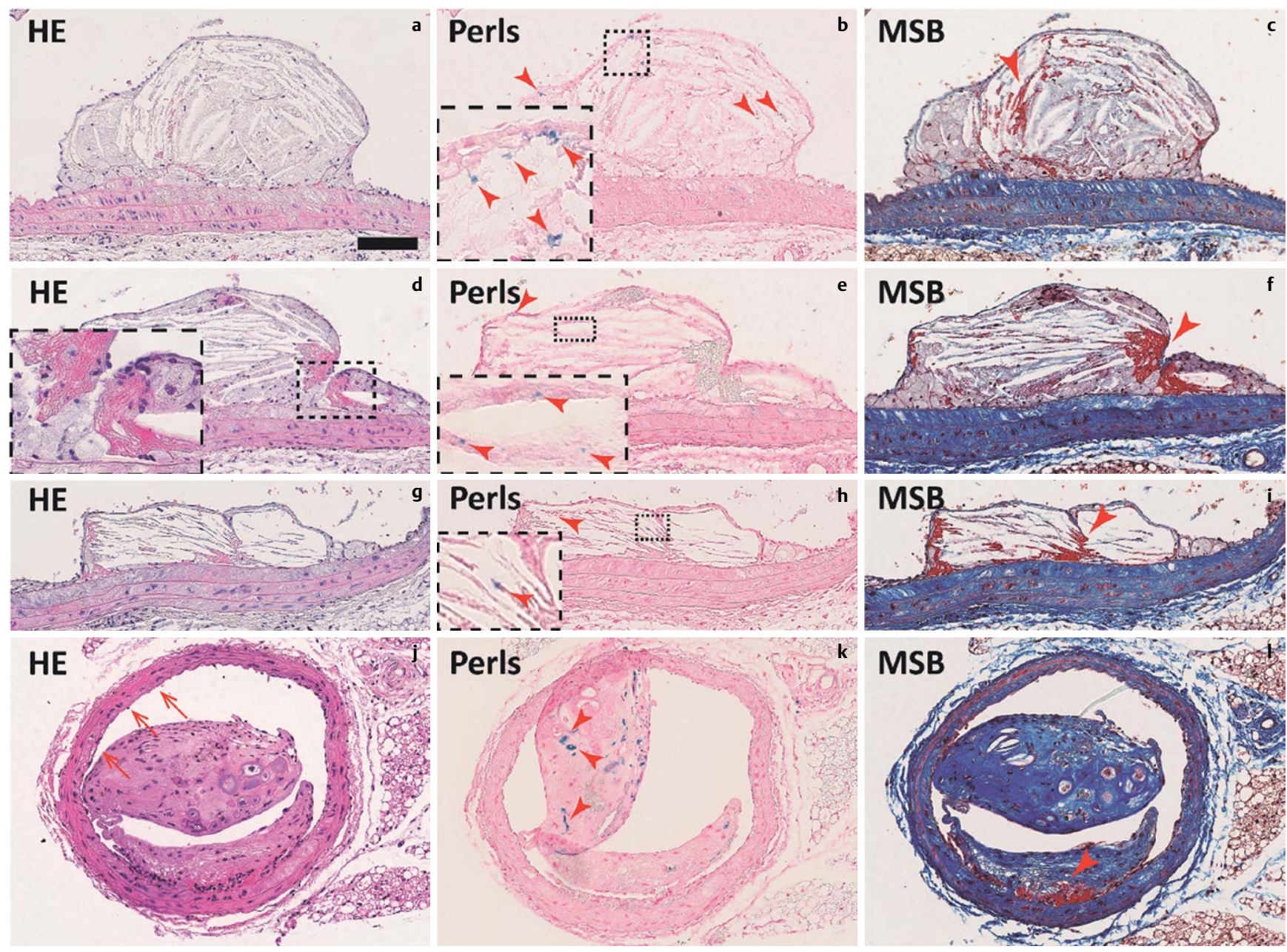

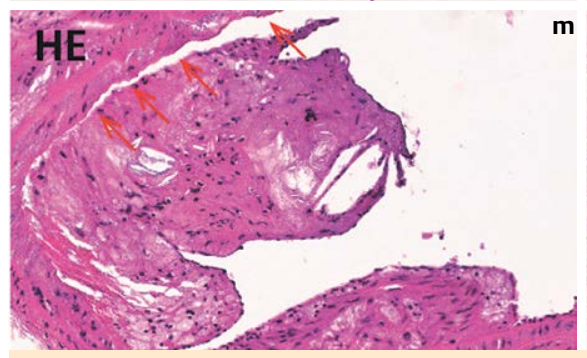

Fig. 2 Plaque disruption in brachiocephalic trunk. (a, d, g, j, m: HE staining; b, e, k, n: Perl's Ferric Iron staining; c, f, i, l, o: MSB staining). Histological analysis of the brachiocephalic trunk revealed plaque in the brachiocephalic artery with iron (blue staining in Perl's indicated by arrowheads in $\mathbf{b}, \mathbf{e}$ and $\mathbf{h}$ ) as a remnant of earlier intraplaque bleeding and fibrin/ fibrinogen deposition (red color in MSB staining; arrowheads in c, $\mathbf{f}$ and $\mathbf{i}$ ). At the bifurcation of the brachiocephalic artery into the right subclavian artery $(\mathbf{m}-\mathbf{o})$ and the right common carotid artery $(\mathbf{j}-\mathbf{I})$, plaques with rem-

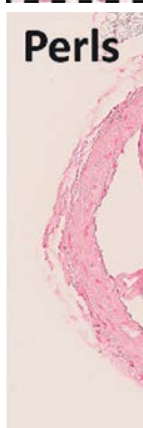

k
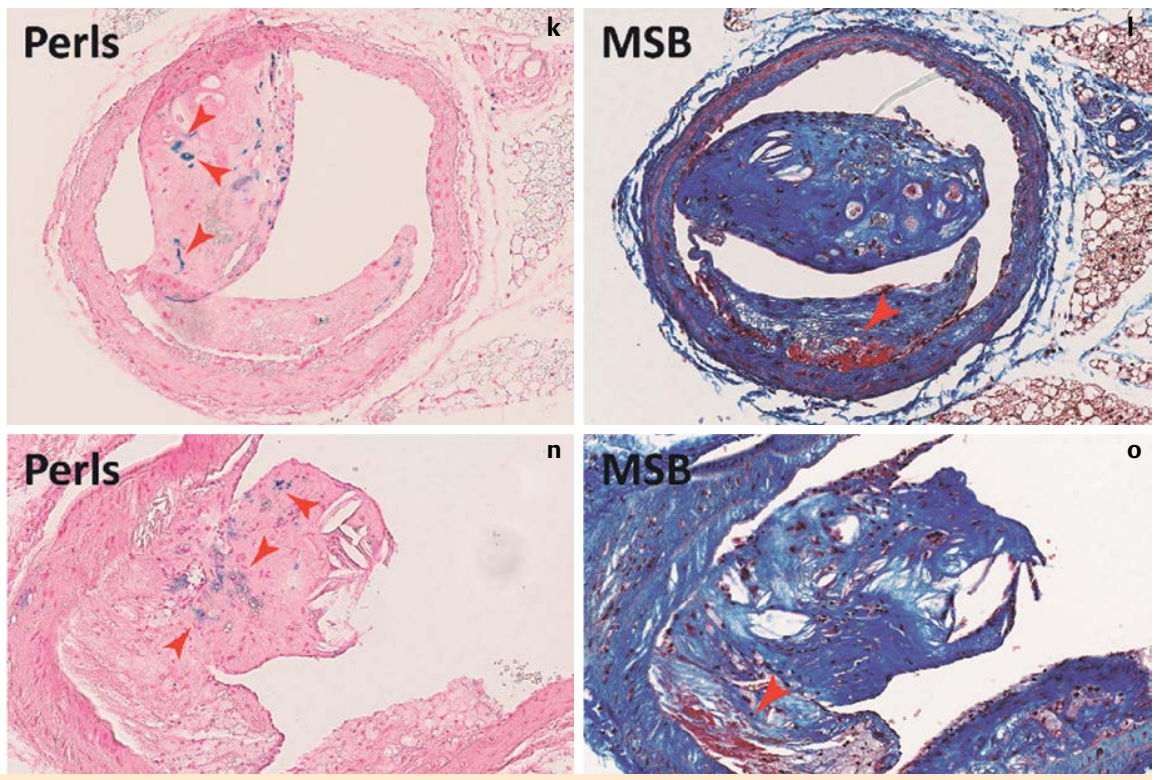

n

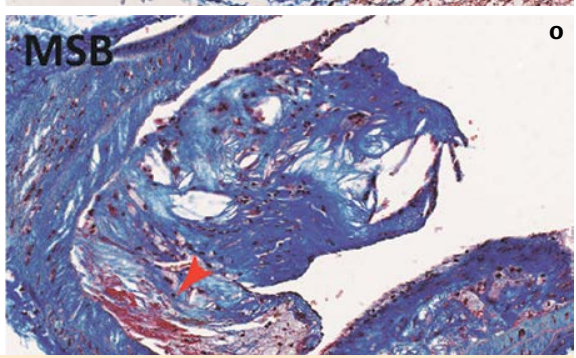

nants of earlier intaplaque bleeding (Perl's Ferric Iron staining; arrowhead in $\mathbf{k}$ and $\mathbf{n}$ ) and fibrin/fibrinogen deposits (red in MSB staining; arrowheads in I and $\mathbf{o}$ ) were present. At both locations dissection of the plaque from the tunica media (indicated by arrows in $\mathbf{j}$ and $\mathbf{m}$ ) was observed, which is more likely to have occurred in vivo rather than due to histological processing, as fibrin/fibrinogen is present at the site where the plaque is still attached to the vessel wall (arrowheads in I and o). Magnification: 800x, scale bar: $100 \mu \mathrm{m}$. 
and the first targeted UCA ( $\bullet$ Fig. 1b, e, Supplementary Video 1). During injection of $\alpha_{v} \beta_{3}$-integrin antibody, 42 min after the first injection, a sudden cessation of flow through the right common carotid artery was observed. Flow cessation coincided with a decrease in right common carotid pulsation followed by vessel constriction ( $\bullet$ Fig. 1f, Supplementary Video 2). Flow cessation in the right common carotid continued for approximately $30 \mathrm{~min}$. At this time, another injection with targeted UCA was performed. During the first $5 \mathrm{~s}$ after arrival of the microbubbles in the aortic arch, hardly any microbubbles passed through the occluded area in the brachiocephalic trunk ( $\odot$ Fig. 1c, Supplementary Video 3). Then, microbubbles slowly started to pass through and reached the right common carotid in the next $10 \mathrm{~s}$. Strikingly, flow through the salivary gland was not restored (० Fig. 1d, Supplementary Video 3).

Histological analysis revealed plaque disruption in 3 advanced atherosclerosis lesions ( $\bullet$ Fig. 2) associated with intraplaque hemorrhage and fibrin/fibrinogen ( $\bullet$ Fig. $\mathbf{2 c}, \mathbf{f}, \mathbf{i}, \mathbf{I}, \mathbf{0}$ ). Plaques with iron deposits (Perl's staining in $\odot \mathbf{F i g ~} \mathbf{2 b}, \mathbf{e}, \mathbf{h}, \mathbf{k}, \mathbf{n}$ ), indicative of remnants of old hemorrhage, and with fibrin/fibrinogen deposits (MSB staining in $\odot \mathbf{~ F i g . ~} \mathbf{2 c}, \mathbf{f}, \mathbf{I}, \mathbf{I}, \mathbf{o}$ ), were present in the brachiocephalic trunk ( $\odot$ Fig. $\mathbf{2 a - i}$ ) and at the bifurcation of the brachiocephalic artery into the right subclavian artery ( $\bullet$ Fig. $\mathbf{2 m - 0}$ ) and the right common carotid artery ( $\bullet$ Fig. $\mathbf{2 j} \mathbf{j} \mathbf{I}$ ). Dissection of the plaque from the tunica media was observed at the bifurcation in both lesions. The presence of fibrin/fibrinogen at the point where the plaque is still connected to the tunic media suggests this dissection occurred in vivo and was not an artifact due to histological processing.

\section{Discussion}

$\nabla$

In a contrast-enhanced ultrasonography study aimed to quantify carotid atherosclerotic neovascularization with microbubble targeted to $\alpha_{\mathrm{v}} \beta_{3}$-integrin in an ApoE $\mathrm{E}^{-/-}$mouse, we observed a live sudden flow cessation which coincided with a decrease in right common carotid pulsation and vessel constriction. Subsequently, we investigated the possible cause for this phenomenon by histological analysis of the affected carotid artery and brachiocephalic trunk. The presence of advanced atherosclerotic lesions in the brachiocephalic trunk, with dissections, points towards a possible plaque rupture as the cause of the observed arterial occlusion. To the best of our knowledge, this is the first study to report a live case of possible plaque rupture in $\mathrm{ApoE}^{-/-}$ mice. We observed dissection and intraplaque hemorrhage, which is a common feature of complex lesions preceding acute ischemic events and is considered a characteristic of previous plaque rupture in mouse models of atherosclerosis [19,23]. However, we did not detect a luminal thrombus or cap rupture, which is a key criterion for plaque rupture in human atherosclerosis. The standard definition of plaque rupture in humans is considered cap disruption of a fibroatheroma with a luminal thrombus communicating with the underlying necrotic core [3]. This definition was extended to animal models [5]. Nevertheless, not all criteria of plaque rupture in humans can be transferred to animals $[24,25]$. A luminal thrombus communicating with a ruptured fibrous cap is very uncommon in $\mathrm{ApoE}^{-/-}$mice, possibly due to a much higher rate of thrombolysis due to low levels of plasma plasminogen activator inhibitor-1 and thrombin-activated fibrinolysis inhibitor in mice compared to humans [15-17]. However, we did detect fibrin/fibrinogen at the base of the plaques.
Besides the possibility that flow was restored because the thrombus resolved, another possibility for the temporary occlusion is embolization of either plaque material or a thrombus. Our observation that flow was not restored in the salivary gland could point to an obstruction distal to the internal right common carotid artery and supports the latter hypothesis.

At the same time, we cannot ignore the possible impact our handling of the animal had on the plaques. Firstly, the animal was exposed to multiple injections, which could have introduced hypervolemia or air bubbles. However, we consider this unlikely as the site of injection was the left jugular vein and at the time of the event only $200 \mu$ l was injected at a slow injection rate. Secondly, we need to consider possible damage generated by UCA in combination with high frequency ultrasound. It has been reported that targeted UCA and high-intensity ultrasound can have destructive effects on microvessels in the rat spinotrapezius muscle [26] and can produce regions of reduced blood flow within tumor microvasculature in mice [27], but none of these effects were shown to occur in larger vessels like the carotid artery. Moreover, at the time of the event no high-intensity ultrasound was applied. Thirdly, the animal was subjected to physical handling, namely slight straightening of the neck and coupling an ultrasound transducer via gel to the neck. This could induce some pressure on the artery although we never observed deformation of the artery during imaging. Also, simultaneous ECG readings excluded a potential cardiac cause for the flow cessation. Finally, the occurrence of the event during antibody injection is more likely to be a coincidence since no plaque disruption induced by antibody injection has been reported.

For the first time, possible spontaneous plaque disruption in the right brachiocephalic artery of an $\mathrm{ApoE}^{-/-}$mouse was observed in real time with contrast-enhanced ultrasonography. Although no thrombus was found in the right carotid artery, plaque dissection from the media and presence of intraplaque hemorrhage in the advanced atherosclerotic lesions, provide the supporting histological evidence for plaque disruption as the possible cause of temporary arterial occlusion.

Affiliations

1 Erasmus Medical Center, Thoraxcenter Biomedical Engineering, Rotterdam, Netherlands

${ }^{2}$ Department of Pathology, Maastricht University, CARIM, Maastricht, Netherlands

${ }^{3}$ Pharmacology \& Toxicology, Maastricht University Medical Center, Maastricht, Netherlands

${ }^{4}$ Lab of Acoustical Wavefield Imaging, Delft University of Technology, Delft, the Netherlands

${ }^{5}$ Pathology, Amsterdam Medical Center, Amsterdam, Netherlands

\section{References}

1 Fitzgerald PJ, Ports TA, Yock PG. Contribution of localized calcium deposits dissection after angioplasty an observational study using intravascular ultrasound. Circulation 1992; 86: 64-70

2 Falk E, Nakano M, Bentzon JF et al. Update on acute coronary syndromes: the pathologists' view. European heart journal 2013; 3410: 719-728

3 Virmani R, Kolodgie FD, Burke AP et al. Lessons from sudden coronary death: a comprehensive morphological classification scheme for atherosclerotic lesions. Arterioscler Thromb Vasc Biol 2000; 20: $1262-1275$

4 Stary HC, Chandler AB, Dinsmore RE. A definition of advanced types of atherosclerotic lesions and a histological classification of atherosclerosis. A report from the committee on vascular lesions of the council on arteriosclerosis, american heart association. Arterioscler Thromb Vasc Biol 1995; 15: 1512-1531

5 Glass CK, Witztum JL. Atherosclerosis. The road ahead Cell 2001; 104: 503-516 
6 Mahoney WM, Schwartz SM. Defining smooth muscle cells and smooth muscle injury. J Clin Invest 2005; 115: 221-224

7 Schwartz SM, Galis ZS, Rosenfeld ME et al. Plaque rupture in humans and mice. Arterioscler Thromb Vasc Biol 2007; 27: 705-713

8 Jackson $C L$. Defining and defending murine models of plaque rupture. Arterioscler Thromb Vasc Biol 2007; 27: 973-977

9 Hansson GK, Heistad DD. Two views on plaque rupture. Arterioscler Thromb Vasc Biol 2007; 27: 697

10 Bentzon JF, Falk E. Atherosclerotic lesions in mouse and man: is it the same disease? Curr Opin Lipidol 2010; 21: 434-440

11 Jawien J. The role of an experimental model of atherosclerosis: apoEknockout mice in developing new drugs against atherogenesis. Curr Pharm Biotechnol 2012; 13: 2435-2439

12 Jin SX, Shen LH, Nie P et al. Endogenous renovascular hypertension combined with low shear stress induces plaque rupture in apolipoprotein E-deficient mice. Arterioscler Thromb Vasc Biol 2012; 32: 2372-2379

13 Falk E, Schwartz SM, Galis ZS et al. Putative murine models of plaque rupture. Arterioscler Thromb Vasc Biol 2007; 27: 969-972

14 Rosenfeld ME, Polinsky P, Virmani $R$ et al. Advanced atherosclerotic lesions in the innominate artery of the apoE knockout mouse. Arterioscler Thromb Vasc Biol 2000; 20: 2587-2592

15 Johnson JL, Jackson CL. The apolipoprotein E knockout mouse: an animal model of atherosclerotic plaque rupture. Atherosclerosis 2001; 154: 399-406

16 Calara F, Silvestre M, Casanada $F$ et al. Spontaneous plaque rupture and secondary thrombosis in apolipoprotein E-deficient and LDL receptordeficient mice. J Pathol 2001; 195: 257-263

17 Zhou J, Moller J, Danielsen CC et al. Dietary supplementation with methionine and homocysteine promotes early atherosclerosis but not plaque rupture in ApoE-deficient mice. Arterioscler Thromb Vasc Biol 2001; 21: 1470-1476
18 Jackson CL, Bennett MR, Biessen EAB et al. Assessment of unstable therosclerosis in mice. Arterioscler Thromb Vasc Biol 2007; 27: 714-720

19 Sasaki T, Kuzuya M, Nakamura $K$ et al. A simple method of plaque rupture induction in apolipoprotein E-deficient mice. Arterioscler Thromb Vasc Biol 2006; 26: 1304-1309

20 Bond AR, Jackson CL. The fat-fed apolipoprotein E knockout mouse brachiocephalic artery in the study of atherosclerotic plaque rupture. J Biomed Biotechnol 2011; 379069

21 Needles A, Arditi M, Rognin NG et al. Nonlinear contrast imaging with an array based micro-ultrasound system. Ultrasound Med Biol 2010; 36: 2097-2106

22 Livingston C. Bancroft's theory and practice of histological techniques. 2008;

23 Von der Thusen JH, van Vlijmen BJ, Hoeben RC et al. Induction of atherosclerotic plaque rupture in apolipoprotein E2/2 mice after adenovirusmediated transfer of p53. Circulation 2002; 105: 2064-2070

24 Hansson GK. Inflammation, atherosclerosis, and coronary artery disease. N Engl J Med 2005; 352: 1685-1695

$25 \mathrm{Ni}$ M, Chen WQ Zhang Y. Animal models and potential mechanisms of plaque destabilisation and disruption. Heart 2009; 95: 1393-1398

26 Skyba DM, Price RJ, Linka AZ et al. Direct in vivo visualization of intravascular destruction of microbubbles by ultrasound and its local effects on tissue. Circulation 1998; 98: 290-293

$27 \mathrm{Hu}$ X, Kheirolomoom A, Mahakian LM et al. Insonation of targeted microbubbles produces regions of reduced blood flow within tumor vasculature. Invest Radiol 2012; 47: 398-405 